ILG-TMP-93-02

April, 1993

\author{
Alexander A.Belov ${ }^{\circledR}$ \\ Int.Inst.for Math.Geophys.and Predictions Theory \\ Warshavskoe sh. 79, k.2 Moscow 113556 Russia
}

Karen D.Chaltikian $\diamond$

Landau Institute for Theoretical Physics

Vorobyevskoe sh.2, Moscow 117940 Russia

\title{
LATTICE ANALOGUE OF W-INFINITY ALGEBRA AND DISCRETE KP-HIERARCHY
}

\begin{abstract}
In development of the started activity on lattice analogues of $W$-algebras, we define the notion of lattice $W_{\infty}$-algebra, accociated with lattice integrable system with infinite set of fields. Various kinds of reduction to lattice $W_{N}$-algebras, related to discrete $N-\mathrm{KdV}$ hierarchies are described. We also discuss the connection of our results with those obtained in the
\end{abstract}

papers of Xiong [13] and Bonora [14].

\section{Introduction}

Recently there has been a great interest in the lattice analogues of $2 \mathrm{D}$ field theory models possessing conformal invariance [1-4]. For the first time this interest arised in connection with Liouville model [1-3]. As is well known, its quantization in continuous limit is rather difficult in the range of central charges $1<c<25$, where usual perturbation theory is inapplicable. 'Latticization' of the world sheet can be viewed as some alternative to usual point-splitting method of regularization. That's why lattice conformal theories give another way of quantization, more convenient in some cases in comparison with that of based on normal ordering procedure. An additional motivation for studying the lattice analogue of conformal invariance comes from the traditional understanding of Integrable Massive models as appropriate deformations of Conformal ones [5-7]. It seems highly desirable that this important concept be formulated on purely lattice language.

In the previous paper [8] authors presented a natural lattice analogue of $W_{3}$-algebra. This algebra has been obtained as $W_{3}$-generalization of the Faddeev-Takhtadjian-Volkov (FTV) algebra [1], which is considered presently as lattice analogue of Virasoro algebra. In the paper [8] we proposed some general method to obtain classical and quantum versions of lattice $W$-algebras $(L W)$, based on Feigin construction of lattice screening charges [9]. This method in principle guarantees the possibility to obtain any $L W$ algebra with finite number of fields with integer spins. Besides, considering such an algebra as second Poisson structure one can easily find the corresponding first structure and a family of involutive integrals of motion. Further on true lattice analogues of $W_{N}$ algebras (second structure) will be referred to as $L W_{N}$ and the corresponding first structure to as $L_{*} W_{N}$. The main drawback of the abovementioned construction is that it does not look to admit straightforward generalization for the case of the infinite number of fields. The aim of present paper is to declare an alternative method of building $L_{*} W_{N}$-algebras, based on certain

\footnotetext{
$\checkmark$ e-mail: mitpan@adonis.ias.msk.su [Belov]

$\diamond$ e-mail: mitpan@adonis.ias.msk.su [Chaltikian], chalt@cpd.landau.free.msk.su
} 
reductions of some guessed ${ }^{1}$ lattice analogue of the first Poisson structure for $W_{\infty}$ and to find its relation with some integrable models.

The detailed plan of the paper is as follows. In Sect.1 we remind the main results of the paper [8], concerning free field representation of $L W_{3}$ algebra, and its bi-hamiltonian structure which led us to the the notion of $L_{*} W_{\infty}$ and give some additional motivations of the work. In Sect.2 we discuss some interesting properties of the Toda Lattice Hierarchy (TLH), which turn out to be closely related with the notion of "lattice conformal symmetry" and certain $L_{*} W_{\infty}$ reductions. In Sect.3 we give the central definition of $L_{*} W_{\infty}$ and $L W_{\infty}$, discuss two various kinds of their reductions to the algebras with finite number of fields. We also compare our results with those of Xiong [13] and Bonora [14]. Sect.4 is devoted to (bi-)hamiltonian structure of the integrable model, possessing $L W_{\infty}$-symmetry and Lax representaion of the model and discuss its connection with both types of reduction. In the Appendix we bring some useful formulas concerning the multihamiltonian structure of TLH.

\section{Acknowledgments}

Authors are greatly indebted to Prof. I.M.Krichever for valuable discussions and very useful remarks. We also thank Prof. A.S.Mezhlumian for the help in preparation of the text for publication.

\section{1. $L W_{3}$-algebra - starting point.}

This section contains some basic definitions and results and can be considered as practical introduction in the subject. We start from Feigin construction of lattice screening vertex operators. Basic lattice variables being the direct analogues of continuum screening charges [10] are defined via their commutation relations as follows:

$$
\begin{gathered}
a_{i}(m) a_{j}(n)=q^{A_{i j}} a_{j}(n) a_{i}(m) \text {, when } n>m \\
\text { and } \\
a_{i}(n) a_{i+1}(n)=q^{-1} a_{i+1}(n) a_{i}(n) \\
a_{i}(n) a_{j}(n)=a_{j}(n) a_{i}(n) \text {, when }|i-j| \geq 2
\end{gathered}
$$

where $A_{i j}$ is Cartan matrix of the appropriate semisimple Lie algebra. Note that $q$ here can be treated as free parameter contrary to the continuous case where it was rigidly determined. Correspondingly, lattice analogues of the screening charges are defined as

$$
Q_{i}=\sum_{n} a_{i}(n)
$$

For the case of $S L_{3}$ we denote basic lattice vertices by $b_{n}$ and $c_{n}$. Further on we will discuss only classical case, the original Poisson brackets (PB) becoming

$$
\begin{gathered}
\left\{b_{n}, b_{m}\right\}=\epsilon(m-n) b_{n} b_{m} \quad\left\{c_{n}, c_{m}\right\}=\epsilon(m-n) c_{n} c_{m} \\
\left\{b_{n}, c_{m}\right\}=\theta(n-m) b_{n} c_{m}
\end{gathered}
$$

where $\epsilon(n)$ and $\theta(n)$ are standardly defined as

$$
\epsilon(n)=\left\{\begin{array}{ll}
1, & \text { if } n>0 \\
0, & \text { if } n=0 \\
-1, & \text { if } n<0
\end{array} \quad \theta(n)= \begin{cases}1, & \text { if } n \geq 0 \\
0, & \text { otherwise }\end{cases}\right.
$$

1 An algebra related to ours via some involutive transformation recently has been obtained in the paper of Xiong [13] . Detailed discussion is given in Sect.3 
Going further in parallel with the continuum limit, Feigin proposed to define Vir and $W$ lattice generators as 3- and 4-point objects lying in the zero-graded part of kernel of the adjoint action of lattice screening charges, the gradation being defined as $\left(\bar{a} \equiv a^{-1}\right)$ :

$$
\operatorname{deg}\left(a_{r}(n)\right)=1 \quad \operatorname{deg}\left(\bar{a}_{r}(n)\right)=-1
$$

Introducing more convenient variables $p_{n}$ and $d_{n}$

$$
p_{n} \equiv \bar{b}_{n} b_{n+1} \quad \text { and } \quad d_{n} \equiv c_{n} \bar{c}_{n+1}
$$

with the local PB's

$$
\begin{array}{ccc}
\left\{p_{n}, p_{n+1}\right\}=p_{n} p_{n+1} & \left\{p_{n}, d_{n}\right\}=p_{n} d_{n} \\
\left\{d_{n}, d_{n+1}\right\}=d_{n} d_{n+1} & \left\{p_{n+1}, d_{n}\right\}=-p_{n+1} d_{n}
\end{array}
$$

after some calculations we obtain the following expressions for bi- and three-local (in terms of original vertices) generators [8]:

$$
\begin{gathered}
L_{n}=\left(p_{n+1} d_{n}+p_{n+1}+d_{n}\right)\left(1+p_{n}+d_{n}\right)^{-1}\left(1+p_{n+1}+d_{n+1}\right)^{-1} \\
W_{n}=d_{n} p_{n+2}\left(1+p_{n}+d_{n}\right)^{-1}\left(1+p_{n+1}+d_{n+1}\right)^{-1}\left(1+p_{n+2}+d_{n+2}\right)^{-1}
\end{gathered}
$$

These fields form closed algebra $L W_{3}[8]$ :

$$
\begin{array}{cc}
\left\{L_{n}, L_{n+1}\right\}=\left(L_{n} L_{n+1}-W_{n}\right)\left(1-L_{n}-L_{n+1}\right) & \left\{L_{n}, L_{n+2}\right\}=-L_{n} L_{n+1} L_{n+2}+W_{n} L_{n+2}+W_{n+1} L_{n} \\
\left\{L_{n}, W_{n}\right\}=-W_{n} L_{n} L_{n+1}+W_{n}^{2}=\left\{W_{n}, L_{n+1}\right\} \\
\left\{W_{n}, L_{n+3}\right\}=-W_{n} L_{n+2} L_{n+3}+W_{n} W_{n+2} & \left\{W_{n}, L_{n+2}\right\}=W_{n} L_{n+2}\left(1-L_{n+1}-L_{n+2}\right)+W_{n} W_{n+1} \\
\left\{L_{n}, W_{n+1}\right\}=L_{n} W_{n+1}\left(1-L_{n}-L_{n+1}\right)+W_{n} W_{n+1} & \left\{L_{n}, W_{n+2}\right\}=-L_{n} L_{n+1} W_{n+2}+W_{n} W_{n+2} \\
\left\{W_{n}, W_{n+1}\right\}=W_{n} W_{n+1}\left(1-L_{n}-L_{n+2}\right) & \left\{W_{n}, W_{n+2}\right\}=W_{n} W_{n+2}\left(1-L_{n+1}-L_{n+2}\right) \\
\left\{W_{n}, W_{n+3}\right\}=-W_{n} W_{n+3} L_{n+2}
\end{array}
$$

In continuous limit, setting $x \equiv n \Delta$

$$
\begin{gathered}
W_{n} \longrightarrow \frac{1}{27}\left(1-\Delta^{2} u(x)-\frac{\Delta^{3}}{2} w(x)\right) \\
L_{n} \longrightarrow \frac{1}{3}\left(1-\frac{\Delta^{2}}{3} u(x)\right)
\end{gathered}
$$

one can easily check that the fields $u(x)$ and $w(x)$ form classical $W_{3}$-algebra.

Indeed, the same path beginning from the single vertex $a_{n}$, can be passed in order to obtain FTV algebra [9], appeared first in the paper on lattice Liouville theory and Volterra model [1]:

$$
\begin{gathered}
\left\{S_{n}, S_{n+1}\right\}=S_{n} S_{n+1}\left(1-S_{n}-S_{n+1}\right) \\
\left\{S_{n}, S_{n+2}\right\}=-S_{n} S_{n+1} S_{n+2}
\end{gathered}
$$

where $S_{n}$ has the following representation $\left(p_{n}=\bar{a}_{n} a_{n+1}\right)[1,2,9]$ :

$$
S_{n}=p_{n+1}\left(1+p_{n}\right)^{-1}\left(1+p_{n+1}\right)^{-1}
$$

Strange as it might seem that the fields $L_{n}$ do not form a subalgebra in $L W_{3}$. However, looking attentively at the first line of commutation relations (3), one can see that setting $W_{n}=0$ one obtains FTV algebra (4). 
The model with symmetry (3), as well as Volterra model [3], turns out to possess multihamiltonian structure [8]. Namely, one can set the following first structure on the space of the fields $L_{n}$ and $W_{n}$, viewing (3) as the second one:

$$
\begin{array}{cc}
\left\{L_{n}, L_{n+1}\right\}_{1}=L_{n} L_{n+1}-W_{n} & \left\{W_{n}, W_{n+1}\right\}_{1}=W_{n} W_{n+1} \\
\left\{L_{n+2}, W_{n}\right\}_{1}=-L_{n+2} W_{n} & \left\{L_{n}, W_{n+1}\right\}_{1}=L_{n} W_{n+1} \\
\left\{W_{n}, W_{n+2}\right\}_{1}=W_{n} W_{n+2}
\end{array}
$$

This algebra will be further referred to as $L_{*} W_{3}$.

One can easily check, that the following are the involutive hamiltonians of the model

$$
\begin{gathered}
\mathcal{H}^{(1)}=\sum_{n} L_{n} \\
\mathcal{H}^{(2)}=\sum_{n}\left(\frac{L_{n}^{2}}{2}+L_{n} L_{n+1}-W_{n}\right) \\
\mathcal{H}^{(3)}=\sum_{n}\left(\frac{L_{n}^{3}}{3}+L_{n}^{2} L_{n+1}+L_{n} L_{n+1}^{2}+L_{n} L_{n+1} L_{n+2}-W_{n}\left(L_{n+1}+L_{n+2}\right)-L_{n}\left(W_{n}+W_{n+1}\right)\right)
\end{gathered}
$$

related by the recursion

$$
\left\{\mathcal{H}^{(i)}, \Psi\right\}_{2}=\left\{\mathcal{H}^{(i+1)}, \Psi\right\}_{1}
$$

where $\Psi$ is any field from the set $\left\{L_{n}, W_{n}\right\}$.

\section{Doubling transformation in reduced TLH.}

In this section we briefly discuss some interesting properties of the well-known TL system $[11,12]$ which turn out to be relevant for the subject involved. We start from the Hamiltonian

$$
\mathcal{H}_{T}=\sum_{n}\left(U_{n}+\frac{p_{n}^{2}}{2}\right)
$$

where $U_{n}=e^{q_{n}-q_{n-1}}$ and Poisson structure is standard

$$
\left\{p_{n}, q_{m}\right\}=\delta_{n, m}
$$

This system possess multihamiltonian structure [11-13], and one can easily obtain the recursion operator and higher Poisson structures (complete derivations are brought in the Appendix). It will be convenient for us to refer to the original PB (7) as $\frac{1}{2}$-bracket. Then the first bracket of TL is given by

$$
\begin{array}{rlrl}
\left\{U_{n}, U_{n+1}\right\} & =-U_{n} U_{n+1} & \left\{p_{n}, p_{n+1}\right\}=-U_{n+1} \\
\left\{U_{n}, p_{n}\right\} & =-U_{n} p_{n} \quad\left\{U_{n+1}, p_{n}\right\}=U_{n+1} p_{n}
\end{array}
$$

Our further interest will be in the "restricted" model with $p_{n}=0$, or in other words, with all "halfinteger" times equal to zero. In this case, as is well-known TL becomes the Volterra model [12]. Through the recursion procedure (A.4) one can find its second structure to be the FTV algebra, which we write here in a more convenient than (4) but equivalent form

$$
\begin{gathered}
\left\{U_{n}, U_{n+1}\right\}_{2}=-U_{n} U_{n+1}\left(U_{n}+U_{n+1}\right) \\
\left\{U_{n}, U_{n+2}\right\}_{2}=-U_{n} U_{n+1} U_{n+2}
\end{gathered}
$$


We note that the canonical FTV algebra can be obtained either as a linear combination of the compatible structures (9) and (8) or as a second structure for the system of hamiltonians, differing from the original ones by some linear transformation.

The recursion procedure provides us also with $\frac{3}{2}$-bracket (see Appendix) but we do not bring it here as it is identical zero on the surface $p_{n}=0$ [13].

Now we describe the doubling transformation of the TLH. For this introduce the notations

$$
u_{n}=U_{2 n} \quad v_{n}=U_{2 n+1}
$$

and define the fields

$$
p_{n}^{(1)} \equiv u_{n}+v_{n} \quad U_{n}^{(1)} \equiv v_{n-1} u_{n}
$$

One readily checks that these fields form algebra (8). Indeed, this "doubling" procedure can be carried on again. One can obtain TLH's for the set of pairs $\left(p_{n}^{(i)}, U_{n}^{(i)}\right)$. Thus, a separate interesting problem of studying this system as a whole arises. However, as this is not the subject of the present paper, we will not elaborate on it here.

Let us consider another realization of the algebra (8) from the "doubled" variables, introducing the generators as

$$
l_{n} \equiv p_{n} \quad w_{n} \equiv u_{n} u_{n+1}+u_{n} v_{n+1}+v_{n} v_{n+1}
$$

The relations have the form

$$
\begin{gathered}
\left\{l_{n}, l_{n+1}\right\}=-l_{n} l_{n+1}+w_{n} \quad\left\{l_{n}, w_{n}\right\}=0 \\
\left\{l_{n}, w_{n+1}\right\}=l_{n+2}\left(w_{n}-l_{n} l_{n+1}\right) \quad\left\{l_{n+2}, w_{n}\right\}=l_{n}\left(l_{n+1} l_{n+2}-w_{n+1}\right) \\
\left\{w_{n}, w_{n+1}\right\}=-\left(l_{n} l_{n+1}-w_{n}\right)\left(l_{n+1} l_{n+2}-w_{n+1}\right) \\
\left\{w_{n}, w_{n+2}\right\}=l_{n} l_{n+3}\left(w_{n+1}-l_{n+1} l_{n+2}\right)
\end{gathered}
$$

This algebra will be obtained in next section from the certain reduction of $L_{*} W_{\infty}$.

\section{Universal algebras $L_{*} W_{\infty}$ and $L W_{\infty}$.}

In this section we give the universal generalization of the first structures (5) and (8) to the case of infinite number of fields with spins running from 2 to $\infty$. This algebra have been called $L_{*} W_{\infty}$ in previous sections. The objects of study in this section will be the integer spin fields (ISF) $\left\{A_{n}^{(p)}\right\}_{p=1}^{\infty}$ with spins $p+1$. We also use formal notations $A_{n}^{(0)} \equiv 1$ and $A_{n}^{(p<0)} \equiv 0$. In examples we will write

$$
\begin{array}{ccc}
A_{n}^{(1)} \equiv A_{n}, & A_{n}^{(2)} \equiv B_{n}, & A_{n}^{(3)} \equiv C_{n}, \\
A_{n}^{(4)} \equiv D_{n}, & A_{n}^{(5)} \equiv E_{n}, \quad \ldots
\end{array}
$$

The name ISF is inspired by the continuous limit in which the fields $A_{n}^{(p)}$ have the following form

$$
\begin{aligned}
& A_{n} \rightarrow 1 \oplus w_{2} \Delta^{2} \oplus O\left(\Delta^{3}\right) \\
& B_{n} \rightarrow 1 \oplus w_{2} \Delta^{2} \oplus w_{3} \Delta^{3} \oplus O\left(\Delta^{4}\right) \\
& C_{n} \rightarrow 1 \oplus w_{2} \Delta^{2} \oplus\left(w_{3} \oplus w_{2}^{\prime}\right) \Delta^{3} \oplus w_{4} \Delta^{4} \oplus O\left(\Delta^{5}\right) \\
& D_{n} \rightarrow 1 \oplus w_{2} \Delta^{2} \oplus\left(w_{3} \oplus w_{2}^{\prime}\right) \Delta^{3} \oplus\left(w_{4} \oplus w_{3}^{\prime} \oplus w_{2}^{\prime \prime} \oplus w_{2}^{2}\right) \Delta^{4} \oplus w_{5} \Delta^{5} \oplus O\left(\Delta^{6}\right)
\end{aligned}
$$


where $O\left(\Delta^{m}\right)$ means the terms irrelevant for the purpose of obtaining the continuos limit and $w_{k}(x)$ are the classical analogues of quantum fields $W^{(p)}(x)$ forming $W_{\infty}$-algebra, $W^{(2)}(x) \equiv T(x)$ being energy-momentum tensor and having the OPE of the form

$$
T(x) W^{(p)}(z)=\frac{p W^{(p)}(z)}{(x-z)^{2}}+\frac{\partial W^{(p)}(z)}{x-z}
$$

\section{3.a. First bracket for the ISF.}

We propose the following natural generalization of the first Poisson structures (5) and (8) for the case of infinite number of fields.

$$
\left\{A_{n}^{(p)}, A_{n+m}^{(q)}\right\}=\theta_{m}^{p, q} \cdot\left(-A_{n}^{(p)} A_{n+m}^{(q)}+A_{n}^{(q+m)} A_{n+m}^{(p-m)}\right)
$$

where $\theta$-function is defined as a sum of $\delta$-functions over the appropriate interval

$$
\theta_{m}^{p, q} \equiv \theta(0 \leq p-m \leq q-1) \equiv \sum_{k=0}^{q-1} \delta_{k, p-m}
$$

Proof of Jacobi identity is straightforward and relies on the three main properties of this $\theta$-function:

$$
\begin{aligned}
& \theta_{m}^{p, q}=\theta_{m+k}^{p+k, q} \\
& \theta_{m-k}^{p, q+k}=\theta_{m}^{p, q}+\theta_{p+1}^{m, k} \\
& \theta_{m+k}^{p, q-k} \delta_{q, k+r}=\theta_{m}^{p, q} \theta_{k}^{p-m, r}-\theta_{k}^{q, r} \theta_{m+k}^{p, q-k}-\theta_{m+k}^{p, r} \theta_{m}^{r+m+k, q}
\end{aligned}
$$

We would like to note also that Jacobi identity for this algebra has the form of classical Yang-Baxter equation with discrete spectral parameter. To see this it is sufficient to rewrite (12) in the form

$$
\left\{A_{n}^{(p)}, A_{m}^{(q)}\right\}=r_{r s}^{p q}(m-n) A_{n}^{(r)} A_{m}^{(s)}
$$

Now we are in a position to define the first type of reduction, which we call $L_{*} W_{N}$. Namely, we simply set the constraints

$$
A_{n}^{(p)}=0, \quad \text { for all } \quad p \geq N
$$

The obtained algebra $L_{*} W_{N}$ defines the first Poisson structure for the lattice analogue of the so-called $N$-th KdV hierarchy. In particular, algebras (5)- $L_{*} W_{3}\left(A_{n} \equiv L_{n}, B_{n} \equiv W_{n}\right)$ and (8) (for $\left.p_{n}=0\right)-L_{*} V i r$ can be obtained in this way. Surely, knowing the algebra $L_{*} W_{N}$ and full set of its Hamiltonians (see next section for explicit expressions), one can easily obtain the true $L W_{N}$-algebra, like FTV (=LVir) and $L W_{3}$, which in continuous limit becomes classical $w_{N}$-algebra, corresponding to $N-\mathrm{KdV}$ integrable sytem. Clearly, this algebra gives the second bracket for $N$-KdV lattice hierarchy. General formula also can be obtained and has the form:

$$
\begin{aligned}
\left\{A_{n}^{(p)}, A_{n+m}^{(q)}\right\}_{2}= & -A_{n}^{(p)} A_{n+m-1}^{(1)} A_{n+m}^{(q)}-A_{n}^{(p)} A_{n+p}^{(1)} A_{n+m}^{(q)} \\
& +A_{n}^{(p)} A_{n+m-1}^{(q+1)}+A_{n}^{(p+1)} A_{n+m}^{(q)} \\
& +A_{n}^{(q+m)} A_{n+m-1}^{(1)} A_{n+m}^{(p-m)}+A_{n}^{(q+m)} A_{n+p}^{(1)} A_{n+m}^{(p-m)} \\
& -A_{n}^{(q+m)} A_{n+m-1}^{(p-m+1)}-A_{n}^{(q+m)} A_{n+m}^{(p-m+1)}
\end{aligned}
$$

but there are some technical difficulties for several particular commutators of the operators sitting on the adjacent sites. These commutators (i.e. with $m=0, \pm 1, p, p \pm 1$ ) are given by slightly different formulas. We do not bring them here for the lack of place; however, for any finite $N$ all the 2-brackets can be easily computed from the bihamiltonian recursion relation, as all the integrals of motion are known for the system (see Sect. 4). 
Ending this paragraph, it is worth noting, that for any $L W_{N}$-algebra with finite $N$ one might expect that all the fields $A_{n}^{(p)}$ should be expanded to $N$-th order in lattice constant; however, as we have established earlier, it is sufficient to consider only first $p$ terms in the expansion of $A^{(p)}$ in order to obtain the desired object $L W_{N}$.

\section{3.b. Second type of reduction and Xiong-Bonora algebra [13,14]}

First of all define the transformation from ISF to the string generating fields (SGF) as follows:

$$
\begin{aligned}
& L_{n, n}=A_{n}^{(1)} \\
& L_{n, n+1}=A_{n}^{(1)} A_{n+1}^{(1)}-A_{n}^{(2)} \\
& L_{n, n+2}=A_{n}^{(1)} A_{n+1}^{(1)} A_{n+2}^{(1)}-A_{n}^{(1)} A_{n+1}^{(2)}-A_{n}^{(2)} A_{n+2}^{(1)}+A_{n}^{(3)} \\
& \text {.. } \ldots \text {. . } \\
& L_{n, n+p-1}=\sum_{\vec{m} \in \mathcal{P}} \prod_{j=0}^{|\vec{m}|-1} A_{n+M_{j}}^{\left(m_{j+1}\right)}(-1)^{p+|\vec{m}|}
\end{aligned}
$$

where $\mathcal{P}$ is the set of partitions of segment $[n, n+p] \cap Z$ and other notations are

$$
\begin{gathered}
\vec{m}=\left(m_{1}, m_{2}, \ldots, m_{s}\right), \quad s=|\vec{m}|, \\
\sum_{l=1}^{s} m_{l}=p, \quad M_{j}=\sum_{l=1}^{j} m_{l}, \quad M_{0}=0
\end{gathered}
$$

The main interesting property of this transformation is that is involutive, i.e. if we denote the r.h.s. of eq.(16) by $\mathcal{F}_{p}\left(\left\{A_{m}^{(q)}\right\}\right)$ than one has

$$
A_{n}^{(p)}=\mathcal{F}_{p}\left(\left\{L_{m, m+q}\right\}\right)
$$

The original first structure (12) induces some 1-bracket on the SGF's and direct calculation shows that bracket to be nothing but Xiong-Bonora algebra (see formula (15) of Ref.13 or formula (3.40) or ref.14) if one identifies our SGF's with the fields from Ref.14 as

$$
L_{n, n+p} \equiv a_{p+1}(n)
$$

Now the reduction which we call $L^{*} W_{N}$ ( $W$ now only reminds us about its origination from $L_{*} W_{\infty}$ ) is defined by setting the constraints on the ISF, which can be very simply written in terms of SGF's (16)

$$
L_{n, n+p}=0 \quad \text { for all } \quad p \geq N
$$

This reduction for $N=2$ gives us an abelian algebra

$$
\left\{A_{n}, A_{m}\right\}=0
$$

In the case of $N=3$ we obtain the algebra (11) $\left(A_{n} \equiv l_{n}, B_{n} \equiv w_{n}\right)$ about which we have known from previous section that it gives TLH 1-bracket written in "doubled" space (10), if one rewrite it in terms of SGF's substituting the expressions (17) instead of $A_{n}^{(p)}$. One should mention here that the name "string generating field" comes from the explicit realization of the $N=3$ case in terms of the "doubled" space variables (10), in which the original ISF's are expressed via the recursion relation

$$
\begin{aligned}
A_{n}^{(p+1)} & =u_{n} A_{n}^{(p)}+v_{n} v_{n+1} \ldots v_{n+p} \\
& =u_{n} u_{n+1} \ldots u_{n+p}+A_{n}^{(p)} v_{n+p}
\end{aligned}
$$


Operating with the SGF's from the very beginning Xiong [13] obtained TLH 1- and $\frac{3}{2}$-brackets and showed that some appropriate combination of those gives two commuting Virasoro algebras in the continuous limit. However, his scheme failed in obtaining true second Poisson structures for $N>3$ ( $L W_{N}$-algebras), and so we believe that an appropriate language here is that of ISF's, where one obtains true first brackets for lattice $N-\mathrm{KdV}$ hierarchies. We believe, in particular, that true lattice Virasoro is an algebra closed on one field, i.e. FTV algebra which dtermines the second Poisson structure of the TLH on the surface $p_{n}=0$, rather than the abovementioned linear combination of its 1 - and $\frac{3}{2}$-structures.

\section{Hamiltonian structures and Lax representation.}

In this section we describe the Lax representation of the integrable system associated with $L W_{\infty}$. Formula for $L$-operator also has been obtained in [13]. Its upper triangular matrix elements are given by the set of SGF's $L_{n, n+p}(16)$, and the lower ones are defined as

$$
L_{n, n-k}=\delta_{k, 1}, \quad k>0
$$

Hamiltonians as usual are given by

$$
\mathcal{H}^{(k)}=\frac{1}{k} \operatorname{Tr}\left(L^{k}\right)
$$

and coincide with those of $N$-KdV hierarchy under the constraints (14).

Again we emphasize the advantage of the ISF's language. In Ref.14 it has been noticed, that equations of motion for $L_{n, n+p}$ in the $N=4$ case on the surface $L_{n, n}=0$ cannot be identified with discrete Boussinesq hiearchy as is naturally expected from the multimatrix models intuition because of wrong continuous limit. We see now, that it is not too surprisingly, because only ISF fields give true realization of the lattice $N$-KdV hierarchy. For example, for $N=3$ Hamiltonians coincide with those of lattice Boussinesq hierachy (see Sect.1) and, correspondingly, equations of motion have true continuous limit [8].

\section{Conclusion}

In conclusion, we have built the integrable system with infinite number of fields. This system possesses first and second compatible Poisson structures, the latter being the lattice analogue of $W_{\infty}$ algebra. Certain reduction of this system to $N<\infty$ of fields gives us first and second Poisson structures of lattice $N$-KdV hierarchies. Phase space of the model in this case can be parametrized by $N$ lattice currents as in the example of Sect. 1. There also exists another reduction, in which, generally all the fields have non-trivial, although as we have seen in Sect.3.b not independent evolutions. The reduction here is revealed in that the phase space of the model is parametrized by only few variables. A good example is $N=3$ case, in which we obtain Toda lattice hierarchy after the substitution (19). It is noteworthy, that in this particular case we deal with the phenomena analogous to that of the so-called two-boson realization of KP hierarchy in continuous limit [15].

\section{Appendix. Multihamiltonian structure of the TLH.}

Below some useful instrumentary concerning TLH is presented. First we introduce the following blockmatrix form of $j$-th symplectic structure:

$$
\Omega_{m n}^{(j)} \equiv\left(\begin{array}{cc}
\left\{p_{m}, p_{n}\right\}_{j} & \left\{p_{m}, u_{n}\right\}_{j} \\
\left\{u_{m}, p_{n}\right\}_{j} & \left\{u_{m}, u_{n}\right\}_{j}
\end{array}\right)
$$


Original symplectic structure $\Omega^{\left(\frac{1}{2}\right)}$ (eq.(7)) in this notation is given by

$$
\Omega_{m n}^{\left(\frac{1}{2}\right)}=\left(\begin{array}{cc}
0 & u_{n}\left(\delta_{n, m}-\delta_{n, m+1}\right) \\
-u_{m}\left(\delta_{m, n}-\delta_{m, n+1}\right) & 0
\end{array}\right)
$$

First Poisson structure (eq.(8)) is

$$
\Omega_{m n}^{(1)}=\left(\begin{array}{cc}
u_{m} \delta_{m, n+1}-u_{n} \delta_{m, n-1} & p_{m} u_{n}\left(\delta_{m, n}-\delta_{m, n-1}\right) \\
p_{n} u_{m}\left(\delta_{m, n+1}-\delta_{m, n}\right) & -u_{m} u_{n}\left(\delta_{m, n-1}-\delta_{m, n+1}\right)
\end{array}\right)
$$

One can give the following definition of the Recursion Operator

$$
\mathcal{R} \equiv \Omega^{(1)} \cdot \Omega^{\left(\frac{1}{2}\right)^{-1}}
$$

with explicit form

$$
\mathcal{R}=\left(\begin{array}{cc}
p_{n} \delta_{k, n} & \delta_{k, n}+\frac{u_{n+1} \theta(n-k+2)-u_{n} \theta(n-k+1)}{u_{k}} \\
u_{n}\left(\delta_{k+1, n}+\delta_{k, n}\right) & p_{n} \delta_{k, n}+\frac{\left(p_{n}-p_{n-1}\right) u_{n} \theta(n-k)}{u_{k}}
\end{array}\right)
$$

By construction the following relation is hold:

$$
\vec{v}^{(j)} \cdot \mathcal{R}=\vec{v}^{\left(j+\frac{1}{2}\right)}
$$

where $\vec{v}^{(j)}$ stands for vector field generated by $j$-th Hamiltonian $v_{n}^{(j)}=\left(\frac{\partial \mathcal{H}^{(j)}}{\partial p_{n}}, \frac{\partial \mathcal{H}^{(j)}}{\partial u_{n}}\right)$ Using the set of the definitions above, one can easily prove the following Proposition

Operator $\mathcal{R}$ defines the hierarchy of compatible Poissson structures

$$
\Omega^{(j)}=\mathcal{R}^{2 j-1} \cdot \Omega^{\left(\frac{1}{2}\right)}
$$

such that

$$
\begin{gathered}
\left\{\mathcal{H}^{(i)},\left(\begin{array}{c}
p_{k} \\
u_{k}
\end{array}\right)\right\}_{j}=\left\{\mathcal{H}^{\left(i^{\prime}\right)},\left(\begin{array}{c}
p_{k} \\
u_{k}
\end{array}\right)\right\}_{j^{\prime}} \quad \text { when } \quad i+j=i^{\prime}+j^{\prime} \\
\left\{\mathcal{H}^{(i)}, \mathcal{H}^{(j)}\right\}_{l}=0 \quad \forall i, j, l
\end{gathered}
$$




\section{References}

1. L. Faddeev, L. Takhtadjan, Lect. Notes in Phys. 246 (1986), 166

2. O. Babelon, Phys.Lett. B238 (1990), 234;

O. Babelon, L. Bonora, Phys.Lett. B253 (1991), 365

3. A. Volkov, Zap.Nauch.Sem.LOMI 150 (1986), 17; ibid. 151 (1987)

24 ;

Theor. Math. Phys. 74 (1988) 135

4. F. Falceto, K. Gawedzki, Lattice Wess-Zumino-Witten models and Quantum Groups,

preprint IHES/P/92/73 (1992)

5. A. Zamolodchikov, JETP Lett. 46 (1987), 160; Adv.St.Pure Math.

19 (1989), 641;

Rev.Math.Phys. 1 (1990), 197

6. O. Babelon, Phys.Lett. B215 (1988), 523

7. H. Braden et al., Nucl.Phys. B338 (1990), 689

8. A. Belov, K. Chaltikian, "Lattice analogues of W-algebras and Classical Integrable Equations",

preprint ILG-TMP-93-01, hep-th/9303166

9. B. Feigin, Talk at September Sankt-Peterburg meeting on Geometry and Physics,

1992

10. V. Fateev, S. Lukyanov, Zh.E.T.P. 94(3) (1988), 23

A. Gerasimov, "Quantum Group Structure in Minimal Models", preprint

ITEP-91-22

(1991);

C. Goméz, G. Sierra, "Quantum Group Symmetry of Rational Conformal

Field Theories", preprint UGVA-DPT-90/04-669 (1990)

11. K. Ueno, K. Takasaki, Adv. Studies in Pure Math. 4 (1984), 1

12. L. Faddeev, L. Takhtadjan," Hamiltonian Aprroach in Soliton Theory" (Springer, Berlin 1987

)

13. C. Xiong, Phys. Lett. bf B279 (1992), 347

14. L. Bonora, C. Xiong, "Multi-matrix models without continuous limit", preprint SISSA-ISAS 211/92/EP, (1992)

15. F. Yu, Y.-S. Wu, Phys. Rev. Lett. 68 (1992), 2996 